\title{
Asymmetric dark matter from spontaneous cogenesis in the supersymmetric standard model
}

\author{
Kohei Kamada* \\ Deutsches Elektronen-Synchrotron DESY, Notkestraße 85, D-22607 Hamburg, Germany \\ Masahide Yamaguchi ${ }^{\dagger}$ \\ Department of Physics, Tokyo Institute of Technology, Tokyo 152-8551, Japan
}

(Received 19 January 2012; published 30 May 2012)

\begin{abstract}
The observational relation between the density of baryon and dark matter in the Universe, $\Omega_{\mathrm{DM}} / \Omega_{B} \simeq$ 5 , is one of the most difficult problems to solve in modern cosmology. We discuss a scenario that explains this relation by combining the asymmetric dark matter scenario and the spontaneous baryogenesis associated with the flat direction in the supersymmetric standard model. A part of baryon asymmetry is transferred to charge asymmetry $D$ that dark matter carries, if a symmetry violating interaction that works at high temperature breaks not only $B-L$ but also $D$ symmetries simultaneously. In this case, the present number density of baryon and dark matter can be same order if the symmetric part of dark matter annihilates sufficiently. Moreover, the baryon number density can be enhanced as compared to that of dark matter if another $B-L$ violating interaction is still in thermal equilibrium after the spontaneous genesis of dark matter, which accommodates a TeV scale asymmetric dark matter model.
\end{abstract}

DOI: 10.1103/PhysRevD.85.103530

PACS numbers: 98.80.Cq, 95.35.+d

\section{INTRODUCTION}

The existence of dark matter (DM) [1,2] and the present baryon asymmetry $[1,3]$ are the most important challenges in modern cosmology since it is quite difficult to accommodate them in the context of the standard model of particle physics (SM). Many attempts to explain them separately have been proposed. For example, thermal relics of weakly interacting massive particles (WIMP) can explain the present dark matter abundance elegantly $[2,4]$. Affleck-Dine (AD) baryogenesis [5,6], baryogenesis through leptogenesis [7], electroweak baryogenesis [8], and so on have been proposed as viable models for baryogenesis. However, the coincidence of the present density parameter of baryons and dark matter, $\Omega_{\mathrm{DM}} / \Omega_{B} \simeq 5$ [1], is extremely difficult to explain. One of the reasons lies in the fact that $C P$ violation is essential for baryogenesis [3] while the thermal relic DM does not need $C P$ violation. Thus, we cannot help but regard such a coincidence as an accident as far as the thermal relic scenario is responsible for the present dark matter abundance. ${ }^{1}$

Recently, an alternative scenario of DM that explains this coincidence dubbed as "asymmetric dark matter (ADM)" [10] has been paid great attention. In this scenario, DM is assumed to be charged under a global symmetry, which guarantees the stability of DM. Asymmetry between DM and "anti"-DM is generated by the same physical origin as baryon asymmetry or transferred from baryon asymmetry generated by baryogenesis mechanism

\footnotetext{
*kohei.kamada@desy.de

'gucci@phys.titech.ac.jp

${ }^{1}$ Very recently, an interesting WIMP scenario [9] was proposed, in which WIMP dark matter annihilation is directly responsible for baryogenesis.
}

[11]. If DM annihilation works sufficiently, the present DM is fully asymmetric so that the DM abundance coincides with its asymmetry. Since the present number densities of baryons and DM are linked (typically almost the same) in this scenario, the coincidence is naturally explained as long as the mass of DM, $M_{\mathrm{DM}}$, is of the order of $\mathrm{GeV}$ scale. ${ }^{2} \mathrm{In}$ fact, the mass of DM is related to the proton mass, $m_{\mathrm{p}}$, as follows:

$$
M_{\mathrm{DM}}=\frac{\Omega_{\mathrm{DM}}}{\Omega_{B}} \frac{n_{B} / s}{n_{\mathrm{DM}} / s} m_{\mathrm{p}}=\mathcal{O}(1-10) m_{\mathrm{p}},
$$

where $n_{B(\mathrm{DM})} / s$ and $\Omega_{B(\mathrm{DM})}$ represent the baryon (DM) number-to-entropy ratio and the energy density parameter of baryon (DM), respectively.

Among many baryogenesis models, spontaneous baryogenesis [13] is an attractive scenario in that it can work even in thermal equilibrium. In this mechanism, a nonvanishing velocity of a scalar field, $\dot{\phi}$, violates $C P T$ invariance of the system and generates an effective chemical potential between baryon and antibaryon through a derivative coupling between the scalar field and the baryon current. When the system is in thermal equilibrium including baryon symmetry breaking interactions, the distribution of baryon and antibaryon differs due to the chemical potential, which implies the generation of baryon asymmetry even without $C P$ violation. Such baryon asymmetry is fixed when the symmetry breaking interaction freezes out. Thus, as long as a slow-rolling scalar field derivatively coupled to the baryon/lepton current, it is easy to realize baryogenesis [14].

\footnotetext{
${ }^{2}$ See, for example, Ref. [12] for other attempts to explain the coincidence.
} 
An ADM model associated with spontaneous baryogenesis dubbed as "spontaneous cogenesis" has been proposed by March-Russell and McCullough recently [15]. They discussed the ADM scenario by introducing a global $U(1)_{X}$ symmetry and a slow-rolling light scalar field, which is assumed to be derivatively coupled to the $X$ current. The asymmetry is generated in the DM sector and transmitted to the visible sector through the mixing operator which mediates $X$ and $B-L$ violating interactions but preserves one of their combinations. The annihilation of the symmetric part of dark matter works well in some region of the parameter space. However, since the spontaneous mechanism works in the DM sector, the origins of not only the DM field but also the light scalar field are unidentified, in particular, the presence of the derivative coupling is simply assumed.

On the other hand, Chiba, Takahashi, and one of the present authors (M. Y.) have pointed out that such a derivative interaction can be naturally realized and spontaneous mechanism works well [16] in the context of flat directions in the minimal supersymmetric standard model (MSSM) [17]. Once a flat direction acquires the vacuum expectation value (VEV), the symmetry possessed by the flat direction, typically a combination of the $B$ and $L$ symmetries, is broken. Then, the Nambu-Goldstone (NG) boson associated with its symmetry breaking is shown to be derivatively coupled to the current. Its slow-roll motion due to an explicit symmetry breaking $A$ term induces the chemical potential of particles charged under the symmetry. Thus, baryon $(B-L)$ asymmetry is spontaneously generated as long as the symmetry breaking/mixing operator, which can be easily introduced as a nonrenormalizable operator in the MSSM sector, is in thermal equilibrium. Moreover, it is shown that this mechanism can work even for a flat direction without $B-L$ charge $^{3}$ thanks to the mixing operator, which is essentially the same introduced later by March-Russell and McCullough in the context of cogenesis.

In this paper, we propose another ADM scenario by use of the MSSM flat direction, in which the associated NG boson derivatively couples to a combination of the $B$ and $L$ currents, and its velocity induces an effective chemical potential. By introducing a DM field, its associated charge $D$, and a nonrenormalizable mixing interaction that violates $B, L$ and $D$ charges simultaneously, dark matter and $B-L$ asymmetries are generated at the same time, which favors dark matter with $\mathrm{GeV}$ scale mass. Moreover, typically speaking, there also exists an interaction violating only a combination of $B$ and $L$ charges in the MSSM sector. Provided that such an interaction freezes out a little

\footnotetext{
${ }^{3}$ For the flat direction with $B-L$ charge, Affleck-Dine mechanism works effectively as well as spontaneous baryogenesis. It should be also noticed that this spontaneous mechanism works for a flat direction with neither baryon nor lepton charge by introducing another charge like the Peccei-Quinn charge [16].
}

after the freeze-out of the mixing interaction, ${ }^{4}$ the baryon number density becomes slightly larger than that of the DM. Thus, our scenario can accommodate DM with the weak-scale mass, which may enable us to easily identify the origin of DM. We also find that the annihilation of the symmetric part of DM works efficiently if we extend our visible sector to the next-to-minimal supersymmetric standard model (NMSSM) [18]. Thus, our scenario is suitable for realizing an ADM model and even accommodates $\mathrm{TeV}$ scale dark matter.

This paper is organized as follows. In the next section, the spontaneous baryogenesis in the MSSM flat direction is briefly reviewed. A general discussion of the spontaneous cogenesis as an ADM model is also made in the context of the MSSM. In Sec. III, we discuss concrete examples of spontaneous cogenesis and investigate realistic parameter range to explain the present baryon and DM abundance simultaneously. We also comment on the annihilation process of symmetric part of DM. Section IV is devoted to our conclusions and a discussion.

\section{SPONTANEOUS COGENESIS IN A FLAT DIRECTION}

\section{A. Review of spontaneous baryogenesis in a flat direction}

First we briefly review the spontaneous baryogenesis mechanism proposed by Cohen and Kaplan [13] and its realization in a flat direction of the supersymmetric standard model [16]. Let us consider an effective Lagrangian in which a scalar field $a$ derivatively couples to the baryon current $J_{B}^{\mu}, 6$

$$
\mathcal{L}_{\text {eff }}=-\frac{\partial_{\mu} a}{M} J_{B}^{\mu} .
$$

Here $M$ is a cutoff scale and the baryon current is given by

$$
\begin{aligned}
J_{B}^{\mu} & =\sum_{m} B_{m} j_{m}^{\mu}, \\
j_{m}^{\mu} & = \begin{cases}\bar{\psi}_{m} \gamma^{\mu} \psi_{m} & \text { for fermions, } \\
i\left(\varphi_{m} \partial_{\mu} \varphi_{m}^{*}-\varphi_{m}^{*} \partial^{\mu} \varphi_{m}\right) & \text { for complex scalar fields, }\end{cases}
\end{aligned}
$$

with $B_{m}$ being the baryon number of the field $\psi_{m}\left(\varphi_{m}\right)$. When a homogeneous scalar field $a$ acquires a

\footnotetext{
${ }^{4}$ This requirement is natural because the latter interaction breaks the symmetry more strongly than the former interaction.

${ }^{5}$ In the case that a light scalar field derivatively couples to the $D$ current as discussed in Ref. [15], such a slight enhancement happens for the number density of dark matter instead of baryon charge, even if we introduce an interaction violating only $D$ charges. In this case, the mass of dark matter must be smaller than $\mathrm{GeV}$.

${ }^{6}$ Here we consider only the baryon symmetry $U(1)_{B}$ for simplicity but it can be straightforwardly extended to the case with another global $U(1)$ symmetry.
} 
nonvanishing classical velocity, $\partial_{\mu} a=(\dot{a}, \mathbf{0})$, the effective Lagrangian (2) reads,

$$
\mathcal{L}_{\text {eff }}=-\sum_{m} \frac{\dot{a}}{M} B_{m} n_{m}=-\frac{\dot{a}}{M} n_{B},
$$

where we have used the fact that the 0-th component of baryon current represents the baryon number density, $\sum_{m} B_{m} j_{m}^{0}=\sum_{m} B_{m} n_{m}=n_{B}$. This effective Lagrangian can be regarded as number density multiplied by the effective chemical potential $\mu_{m} \equiv-\dot{a} B_{m} / M$. If there is a baryon number violating interaction and the Universe is hot enough for the interaction to be in thermal equilibrium, fields $\psi_{m}\left(\varphi_{m}\right)$ with baryonic charges are distributed according to their chemical potentials, which leads to the generation of baryon asymmetry in the Universe. Note that this process does not require the Sakharov's condition. Instead, a nonvanishing classical value of $\dot{a}$ breaks the $C P T$ invariance since $\dot{a}$ is odd under the $C P T$ transformation, which spontaneously generates baryon asymmetry.

As the temperature of the Universe decreases, the baryon number violating interaction decouples at $T=T_{\mathrm{dec}}$. If it happens before the decay of the field $a$, the baryon number density is frozen out at the value,

$$
n_{B}\left(t_{\mathrm{dec}}\right)=\sum_{m} B_{m} \frac{g_{m} \kappa_{m} T_{\mathrm{dec}}^{3}}{6}\left(\frac{\mu_{m}}{T_{\mathrm{dec}}}+\mathcal{O}\left[\left(\frac{\mu_{m}}{T_{\mathrm{dec}}}\right)^{3}\right]\right),
$$

where $g_{m}$ is the degree of freedom of $\psi_{m}\left(\varphi_{m}\right)$ and $\kappa_{m}$ is defined as

$$
\kappa_{m}= \begin{cases}1 & \text { for fermions }\left(\psi_{m}\right), \\ 2 & \text { for scalar fields }\left(\varphi_{m}\right)\end{cases}
$$

The baryon-to-entropy ratio is fixed at reheating if $T_{\mathrm{dec}}>$ $T_{R}$ and at the decoupling temperature if $T_{R}>T_{\mathrm{dec}}$, where $T_{R}$ is the reheating temperature. Before reheating, the Universe is dominated by the inflaton oscillation, but thermal plasma has already existed, during which the temperature is related to the Hubble parameter as $T \simeq\left(H M_{G} T_{R}^{2}\right)^{1 / 4}$ [4] with $M_{G}$ and $H$ being the reduced Planck mass and the Hubble parameter, respectively. Thus, the present baryonto-entropy ratio is given by

$$
\frac{n_{B}}{s} \simeq \begin{cases}\frac{15}{4 \pi^{2} g_{* s}} \sum_{m} B_{m} \frac{g_{m} \kappa_{m} \mu_{m}}{T_{\mathrm{dec}}} & \text { for } T_{\mathrm{dec}}<T_{R}, \\ \frac{15}{4 \pi^{2} g_{* s}} \sum_{m} B_{m} \frac{g_{m} \kappa_{m} \mu_{m}}{T_{\mathrm{dec}}}\left(\frac{T_{R}}{T_{\mathrm{dec}}}\right)^{5} & \text { for } T_{\mathrm{dec}}>T_{R},\end{cases}
$$

where $g_{* s} \simeq 200$ is the effective degrees of freedom of relativistic fields. No entropy production after reheating is also assumed.

Following the discussion of Ref. [16], we see how the effective Lagrangian like Eq. (2) naturally arises and spontaneous baryogenesis is realized in the context of the supersymmetric standard model. In supersymmetric theories, there are many flat directions along which the scalar potential vanishes. Since a flat direction is charged under the $U(1)_{B}$ and/or $U(1)_{L}$ symmetries, such symmetries are spontaneously broken if scalar fields acquire nonvanishing expectation values along the flat direction. Then, the NG boson associated with this symmetry breaking derivatively couples to its currents, which is exactly the effective interaction we want. More concretely, a flat direction can be parameterized by composite holomorphic gauge-invariant polynomials as

$$
X=\prod_{i=1}^{N} \chi_{i}
$$

where $N$ is the number of superfields $\chi_{i}$ that constitute the flat direction. The expectation value of a scalar field corresponding to $\chi_{i}$ (we use the same symbol for a superfield and its scalar part) can be decomposed as

$$
\left\langle\chi_{i}\right\rangle=\frac{f_{i}}{\sqrt{2}} e^{i \theta_{i}},
$$

where $f_{i} / \sqrt{2}$ is the absolute value of $\chi_{i}$ and $\theta_{i}$ is its phase. Note that their values are related each other due to the $F$ and $D$-flat conditions. Since the field $\chi_{i}$ is charged under the $U(1)_{B}$ or $U(1)_{L}$ symmetry, we need to treat both symmetries adequately for spontaneous baryogenesis in a flat direction. For this purpose, we consider $U(1)_{A^{ \pm}}$symmetries, which are the two independent linear combinations of the $U(1)_{B}$ and $U(1)_{L}$ symmetries. The $U(1)_{A^{ \pm}}$ charges of the field $\chi_{i}$ are defined as

$$
\begin{gathered}
Q_{i}^{+}=B_{i} \cos \xi+L_{i} \sin \xi \quad \text { for } U(1)_{A^{+}}, \\
Q_{i}^{-}=-B_{i} \sin \xi+L_{i} \cos \xi \quad \text { for } U(1)_{A^{-}},
\end{gathered}
$$

with $\xi$ given by

$$
\tan \xi \equiv \frac{\sum_{i=1}^{N} L_{i}}{\sum_{i=1}^{N} B_{i}}
$$

Here we denote the charges of $\chi_{i}$ as $B_{i}$ and $L_{i}$, respectively. The flat direction $X$ is charged under $U(1)_{A^{+}}$but is not charged under $U(1)_{A^{-}}$as a whole.

In order to identify NG bosons, we express the phase of the scalar field $\theta_{i}$ as $[19,20]$

$$
\theta_{i}=B_{i} \alpha_{B}+L_{i} \alpha_{L}=Q_{i}^{+} \alpha_{+}+Q_{i}^{-} \alpha_{-},
$$

where $\alpha_{B}, \alpha_{L}, \alpha_{+}$and $\alpha$ - are the angles conjugate to the generators of the symmetries, respectively, and are related as

$$
\left(\begin{array}{l}
\alpha_{B} \\
\alpha_{L}
\end{array}\right)=\left(\begin{array}{cc}
\cos \xi & -\sin \xi \\
\sin \xi & \cos \xi
\end{array}\right)\left(\begin{array}{l}
\alpha_{+} \\
\alpha_{-}
\end{array}\right) .
$$

The NG bosons associated with the spontaneous breaking of the $U(1)_{A^{ \pm}}$symmetries are denoted as $a_{ \pm}$and can be expressed by the angles $\alpha_{ \pm}$through the decay constant matrix $F$, 


$$
\left(\begin{array}{l}
a_{+} \\
a_{-}
\end{array}\right)=F\left(\begin{array}{c}
\alpha_{+} \\
\alpha_{-}
\end{array}\right)
$$

Since the (canonical) kinetic terms of $a_{ \pm}$are given by those of $\chi_{i}$ with $f_{i}$ fixed, the decay constant matrix $F$ satisfies the following relation:

$$
F^{T} F=\sum_{i} f_{i}^{2}\left(\begin{array}{cc}
Q_{i}^{+} Q_{i}^{+} & Q_{i}^{+} Q_{i}^{-} \\
Q_{i}^{-} Q_{i}^{+} & Q_{i}^{-} Q_{i}^{-}
\end{array}\right) .
$$

On the other hand, the decay constant matrix $F$ takes the following form because only the NG boson $a_{+}$is lifted by an $A$ term, as we will see later:

$$
F=\left(\begin{array}{cc}
v_{a} & 0 \\
f_{01} & f_{11}
\end{array}\right)
$$

Combining Eqs. (16) and (17) yields the decay constants,

$$
\begin{aligned}
& v_{a}^{2}=\sum_{i} f_{i}^{2} Q_{i}^{+2}-\frac{\left(\sum_{i} f_{i}^{2} Q_{i}^{+} Q_{i}^{-}\right)^{2}}{\sum_{i} f_{i}^{2} Q_{i}^{-2}}, \\
& f_{01}=\frac{\sum_{i} f_{i}^{2} Q_{i}^{+} Q_{i}^{-}}{\sqrt{\sum_{i} f_{i}^{2} Q_{i}^{-2}}}, \quad f_{11}=\sqrt{\sum_{i} f_{i}^{2} Q_{i}^{-2}} .
\end{aligned}
$$

A flat direction is often parameterized by a representative scalar field $\Phi$ as

$$
X=\Phi^{N},
$$

where

$$
\begin{aligned}
& \Phi=\frac{\phi}{\sqrt{2}} e^{i \theta}, \quad \phi \equiv\left(\prod_{i} f_{i}\right)^{1 / N}, \\
& \theta \equiv \frac{1}{N} \sum_{i} \theta_{i}=\frac{a_{+}}{N v_{a}} Q^{+}
\end{aligned}
$$

with $Q^{+} \equiv \sum_{i} Q_{i}^{+}= \pm \sqrt{\left(\sum_{i} B_{i}\right)^{2}+\left(\sum_{i} L_{i}\right)^{2}}$ depending on the sign of $\cos \xi$. Notice that $a_{-}$does not appear here because the flat direction is not charged under the $U(1)_{A^{-}}$ symmetry as a whole.

Now we derive a derivative coupling of $a_{+}$and the $U(1)_{A^{+}}$current as a consequence of the spontaneous breaking of the $U(1)_{A^{+}}$symmetry due to the nonvanishing expectation values of scalar fields along a flat direction.? Under the infinitesimal $U(1)_{A^{+}}$transformation, the NG boson $a_{+}$transforms as $a_{+} \rightarrow a_{+}+v_{a} \epsilon$, where $\epsilon$ is the infinitesimal transformation parameter. At the same time, a matter field $\chi_{m}$ that has a $U(1)_{A^{+}}$charge $Q_{m}^{+}$transforms as $\chi_{m} \rightarrow \chi_{m}+\epsilon \delta \chi_{m}$ with $\delta \chi_{m}=i Q_{m}^{+} \chi_{m}$. Then, the $U(1)_{A^{+}}$ current defined as

\footnotetext{
${ }^{7}$ There is also a derivative coupling between $a_{-}$and the $U(1)_{A^{-}}$current, but $\partial_{\mu} a_{-}$does not acquire a nonvanishing classical value because it remains massless even with the presence of an $A$ term.
}

$$
J_{A^{+}}^{\mu} \equiv-\sum_{m^{\prime}} \frac{\partial \mathcal{L}}{\partial\left(\partial_{\mu} \chi_{m^{\prime}}\right)} \delta \chi_{m^{\prime}}
$$

where $m^{\prime}$ runs all the fields that are charged under the $U(1)_{A^{+}}$symmetry, reduces to

$$
J_{A^{+}}^{\mu}=v_{a} \partial^{\mu} a_{+}+\sum_{m} Q_{m}^{+} j_{m}^{\mu} .
$$

Current conservation gives the equation of motion for $a_{+}$,

$$
\partial_{\mu} J_{A^{+}}^{\mu}=v_{a} \partial^{2} a_{+}+\sum_{m} Q_{m}^{+} \partial_{\mu} j_{m}^{\mu}=0 .
$$

While the first term in the middle equation in Eq. (23) represents the kinetic term for the NG mode $a_{+}$, the second term comes from the following effective Lagrangian:

$$
\mathcal{L}_{\text {eff }}=-\sum_{m} \frac{Q_{m}^{+}}{v_{a}}\left(\partial_{\mu} a_{+}\right) j_{m}^{\mu},
$$

which represents a derivative coupling of the NG boson $a_{+}$ and the charged fields. Thus, a nonvanishing velocity of $a_{+}$ can lead to spontaneous genesis of $U(1)_{A^{+}}$asymmetry as long as the symmetry breaking operator is in thermal equilibrium. When the field $a_{+}$is homogeneous, the effective Lagrangian reduces to $\mathcal{L}_{\text {eff }}=\sum_{m} \bar{\mu}_{m} n_{m}$ with the chemical potential $\bar{\mu}_{m}$ given by

$$
\bar{\mu}_{m} \equiv-\frac{Q_{m}^{+}}{v_{a}} \dot{a}_{+} .
$$

Next, let us discuss the dynamics of a flat direction. Though the scalar potential vanishes along the flat direction in the supersymmetric and renormalizable limit, it can be lifted [6] by the supersymmetry (SUSY)-breaking effects and a nonrenormalizable superpotential of the form,

$$
W_{\mathrm{NR}}=\frac{X^{k}}{N k M_{*}^{N k-3}}=\frac{\Phi^{n}}{n M_{*}^{n-3}},
$$

with $M_{*}$ being a cutoff scale and $n=N k$. Then, the resultant scalar potential is written as [6]

$$
V=V_{\not}+\left(a_{3 / 2} \frac{m_{3 / 2}}{n M_{*}^{n-3}} \Phi^{n}+\text { H.c. }\right)+\frac{|\Phi|^{2 n-2}}{M_{*}^{2 n-6}} .
$$

Here $V_{\not}$ represents the soft breaking effect that depends on the SUSY-breaking mechanism, and the second term in the right-hand side comes from supergravity effects and is called an $A$ term. $m_{3 / 2}$ is the gravitino mass and $a_{3 / 2}$ is a complex numerical factor whose amplitude is of the order of unity. ${ }^{8}$

During inflation, a flat direction can acquire negative Hubble induced mass squared if it has a noncanonical interaction to the inflaton in the Kähler potential with appropriate sign and magnitude [6],

\footnotetext{
${ }^{8}$ Since $a_{3 / 2}$ can be real by field redefinition, we treat it as a real parameter.
} 


$$
V_{H}=-c_{H} H^{2}|\Phi|^{2},
$$

where $c_{H}$ is a positive constant of the order of unity. This term destabilizes the flat direction from the origin so that it acquires a large expectation value, whose magnitude is determined by the balance between the negative Hubble induced mass term and the $F$ term,

$$
\phi_{\min } \simeq\left(H M_{*}^{n-3}\right)^{1 /(n-2)} .
$$

During the inflaton oscillation dominated era, in addition to the Hubble induced mass (28) which remains until the inflaton decay (that is, reheating), thermal effects [21] can also kick the flat direction from the origin through the negative thermal logarithmic potential [22], ${ }^{9}$

$$
V_{\text {thlog }}=-\alpha T^{4} \log \left(\frac{|\Phi|^{2}}{T^{2}}\right) .
$$

This term represents a two-loop finite temperature effect coming from the running of the gauge/Yukawa coupling associated with the nonzero expectation value of the flat direction. Though the sign of $\alpha$ depends on a flat direction, we take it to be positive here. Depending on which term dominates, the flat direction sits on the value given in (29) or the minimum given by

$$
\phi_{\min } \simeq\left(\alpha^{(1 / 2)} T^{2} M_{*}^{n-3}\right)^{1 /(n-1)} .
$$

As the Hubble parameter and the temperature of the Universe decrease, the soft SUSY-breaking mass term and other zero temperature terms overwhelm these terms, which makes the potential minimum the origin. The radial component of the flat direction, $\phi$, starts its oscillation around the origin when $H^{2} \lesssim \partial^{2} V / \partial \phi^{2}$. In the same way, the NG boson also starts its oscillation when

$$
H^{2} \lesssim V^{\prime \prime} \text {, }
$$

where $V^{\prime \prime}$ represents the second derivative of the scalar potential with respect to $a_{+}$. The oscillation of the NG boson $a_{+}$typically takes place earlier than that of $\phi$. Until the onset of its oscillation, the NG boson remains in the slow-roll regime, which is required for the spontaneous genesis [20].

The motion of the NG boson $a_{+}$is governed by an $A$ term because it breaks the $U(1)_{A^{+}}$symmetry explicitly and generates the potential for $a_{+}$. The $A$ term can be expressed as

$$
V_{A}=\frac{a_{3 / 2} m_{3 / 2}}{2^{(n / 2)-1} n M_{*}^{n-3}} \phi^{n} \cos [n \theta]=M_{A}^{4} \cos \left[\frac{k Q^{+}}{v_{a}} a_{+}\right],
$$

with $M_{A}^{4} \equiv \phi^{n} a_{3 / 2} m_{3 / 2} /\left(2^{n / 2-1} n M_{*}^{n-3}\right)$. Then, the equation of motion of $a_{+}$is given by

\footnotetext{
${ }^{9}$ Even during the inflaton oscillation dominated era, thermal plasma exists as a subdominant component of the Universe, coming from the inflaton decay.
}

$$
\ddot{a}_{+}+3 H \dot{a}_{+}-M_{A}^{4} \frac{k Q^{+}}{v_{a}} \sin \left[\frac{k Q^{+}}{v_{a}} a_{+}\right]=0 .
$$

While the condition $V^{\prime \prime} \simeq m_{3 / 2} M_{A}^{4} / \phi_{\min }^{2} \lesssim H^{2}$ is met, the NG boson $a_{+}$is in the slow-roll regime, which leads to

$$
3 H \dot{a}_{+}+M_{A}^{4} \frac{k Q^{+}}{v_{a}} \simeq 0 .
$$

Here we have approximated $\sin \left(k Q^{+} a_{+} / v_{a}\right) \simeq 1$ and $\phi \simeq$ $\phi_{\min }$. Then, $\dot{a}_{+}$acquires a nonvanishing expectation value,

$$
\left|\dot{a}_{+}\right| \simeq \frac{k Q^{+}}{H v_{a}} M_{A}^{4}
$$

For successful spontaneous baryogenesis, the $B$ - $L$ breaking interaction must be decoupled during the slow-roll of the NG boson $a_{+}$because, otherwise, the resulting $B-L$ asymmetry is severely suppressed [20].

Let us see how spontaneous baryogenesis works in a flat direction. Since the sphaleron process is expected to work at a later epoch, ${ }^{10} B-L$ asymmetry must be generated for successful baryogenesis. Otherwise, the sphaleron process would wash out both $B$ and $L$ asymmetries. For this purpose, we consider a $B-L$ breaking interaction, whose amount of $B$ and $L$ violations are denoted as $\Delta_{B}$ and $\Delta_{L}$, respectively. Since $B$ and $L$ asymmetries are generated only through this interaction, they must satisfy the following relation:

$$
\frac{n_{B}}{\Delta_{B}}=\frac{n_{L}}{\Delta_{L}} .
$$

However, the asymmetries based on the chemical potential $\bar{\mu}_{m}$ defined in Eq. (25) do not necessarily obey the above constraint, which requires the chemical potential to be modified. The constraint (37) is rewritten in terms of the number density of $\chi_{m}, n_{m}$, as

$$
\sum_{m} \Xi_{m} n_{m}=0, \quad \Xi_{m} \equiv B_{m} \Delta_{L}-L_{m} \Delta_{B},
$$

which is also equivalent to the condition for the chemical potential for $\chi_{m}, \mu_{m}$,

$$
\sum_{m} \kappa_{m} g_{m} \Xi_{m} \mu_{m}=0
$$

Thus, $\bar{\mu}_{m}$ should be modified so as to be perpendicular to $\Xi_{m}$, yielding the physical chemical potential $\mu_{m}$,

$$
\mu_{m}=\bar{\mu}_{m}-\frac{(\bar{\mu} \cdot \Xi)}{\Xi^{2}} \Xi_{m}
$$

where we have defined the following shorthand:

\footnotetext{
${ }^{10}$ The sphaleron process is usually assumed to be in thermal equilibrium for $T \lesssim 10^{12} \mathrm{GeV}$. However, in case that the weak gauge bosons become massive due to the large VEV of a flat direction, the sphaleron configuration is not excited so that it is effective only after the decay of the flat direction.
} 


$$
Y^{2} \equiv \sum_{m} \kappa_{m} g_{m} Y_{m}^{2}, \quad Y \cdot Z \equiv \sum_{m} \kappa_{m} g_{m} Y_{m} Z_{m} .
$$

Since the number density of $\chi_{m}, n_{m}$, at the decoupling temperature is expressed as

$$
n_{m}\left(T_{\mathrm{dec}}\right)=\frac{\kappa_{m} g_{m}}{6} \mu_{m} T_{\mathrm{dec}}^{2},
$$

the resultant $B$ - $L$ asymmetry is given by

$$
\begin{aligned}
n_{B-L}\left(T_{\mathrm{dec}}\right)= & \sum_{m}\left(B_{m}-L_{m}\right) n_{n}\left(T_{\mathrm{dec}}\right) \\
= & \left(\Delta_{B}-\Delta_{L}\right)\left(\mu_{B} \Delta_{B}+\mu_{L} \Delta_{L}\right) \\
& \times \frac{B^{2} L^{2}}{B^{2} \Delta_{L}^{2}+L^{2} \Delta_{B}^{2}} \frac{T_{\mathrm{dec}}^{2}}{6} .
\end{aligned}
$$

Here we have defined

$$
\mu_{B} \equiv-\frac{\dot{a}_{+} \cos \xi}{v_{a}}, \quad \mu_{L} \equiv-\frac{\dot{a}_{+} \sin \xi}{v_{a}},
$$

and assumed $B_{m} L_{m}=0$ for all the $\chi_{m}$ fields, which are reasonable for the (supersymmetric) standard model particles. From (43), it is manifest that the following two conditions must be satisfied for successful $B$ - $L$ genesis:

$$
\Delta_{B}-\Delta_{L} \neq 0, \quad \mu_{B} \Delta_{B}+\mu_{L} \Delta_{L}=-\Delta_{Q^{+}} \frac{\dot{a}_{+}}{v_{a}} \neq 0 .
$$

Thus, the $B-L$ asymmetry is generated even if a flat direction $X$ itself does not have a $B-L$ charge. Depending on whether the $B-L$ breaking interaction is decoupled before or after the reheating, the final $B$ - $L$ asymmetry is estimated as

$$
\begin{aligned}
\frac{n_{B-L} \simeq}{s} \simeq & \frac{15}{4 \pi^{2} g_{* s}} \\
& \times \frac{\left(\Delta_{B}-\Delta_{L}\right)\left(\mu_{B}\left(T_{\mathrm{dec}}\right) \Delta_{B}+\mu_{L}\left(T_{\mathrm{dec}}\right) \Delta_{L}\right) B^{2} L^{2}}{T_{\mathrm{dec}}\left(\Delta_{B}^{2} L^{2}+\Delta_{L}^{2} B^{2}\right)} \\
& \times \begin{cases}1 & \text { for } T_{\mathrm{dec}}<T_{R}, \\
\left(\frac{T_{R}}{T_{\mathrm{dec}}}\right)^{5} & \text { for } T_{\mathrm{dec}}>T_{R} .\end{cases}
\end{aligned}
$$

After the decay of the flat direction, a part of the $B-L$ asymmetry is converted to the baryon asymmetry through the sphaleron effect,

$$
\frac{n_{B}}{s}=\frac{8}{23} \frac{n_{B-L}}{s} .
$$

Thus, we have a successful baryogenesis scenario with appropriate parameter choices such as reheating temperature or gravitino mass in the context of the supersymmetric standard model [16].

\section{B. Spontaneous cogenesis}

We present a scenario of spontaneous cogenesis in the supersymmetric flat directions in the similar way as dis- cussed in the previous subsection. For this purpose, we introduce an additional $U(1)$ symmetry denoted as $U(1)_{D}$, which is responsible for the stability of dark matter, and a pair of chiral supermultiplets $\Psi$ and $\bar{\Psi}$ whose $U(1)_{D}$ charge are 1 and -1 , respectively. These fields construct a mass term,

$$
W_{\text {mass }}=M_{\Psi} \Psi \bar{\Psi} .
$$

We also assume that the standard model fields are neutral under $U(1)_{D}$ and the superpotential of the system can be expressed as $W=W_{\text {MSSM }}+W_{\text {mass }}$ in the renormalizable limit, where $W_{\text {MSSM }}$ is the superpotential for the MSSM.

\section{The case with only a $B-L-D$ mixing interaction}

Let us first consider a case that there is only a $B-L-D$ mixing interaction. Denoting the amount of $B, L$ and $D$ violation as $\Delta_{B}, \Delta_{L}$ and $\Delta_{D}$ for this interaction, produced asymmetries must satisfy the following relations:

$$
\frac{n_{B}}{\Delta_{B}}=\frac{n_{L}}{\Delta_{L}}=\frac{n_{D}}{\Delta_{D}},
$$

which are equivalent to relations on the number densities of $m$-th field,

$$
\sum_{m} \Xi_{m}^{(1)} n_{m}=\sum_{m} \Xi_{m}^{(2)} n_{m}=0,
$$

with

$$
\Xi_{m}^{(1)} \equiv D_{m} \Delta_{L}-L_{m} \Delta_{D}, \quad \Xi_{m}^{(2)} \equiv D_{m} \Delta_{B}-B_{m} \Delta_{D} .
$$

These relations are also rewritten in terms of chemical potential as

$$
\Xi^{(1)} \cdot \mu=\Xi^{(2)} \cdot \mu=0 .
$$

Then, the physical chemical potential of $m$-th field can be expressed as

$$
\mu_{m}=\bar{\mu}_{m}-\beta_{1} \Xi_{m}^{(1)}-\beta_{2} \Xi_{m}^{(2)},
$$

with the numerical parameters $\beta_{1}$ and $\beta_{2}$ given by

$$
\begin{aligned}
& \beta_{1}=\frac{-\Delta_{B}^{2} \mu_{L} L^{2} D^{2}-\Delta_{D}^{2} \mu_{L} L^{2} B^{2}+\Delta_{B} \Delta_{L} \mu_{B} B^{2} D^{2}}{\Delta_{D}\left(\Delta_{B}^{2} L^{2} D^{2}+\Delta_{L}^{2} D^{2} B^{2}+\Delta_{D}^{2} B^{2} L^{2}\right)}, \\
& \beta_{2}=\frac{-\Delta_{L}^{2} \mu_{B} B^{2} D^{2}-\Delta_{D}^{2} \mu_{B} B^{2} L^{2}+\Delta_{B} \Delta_{L} \mu_{L} L^{2} D^{2}}{\Delta_{D}\left(\Delta_{B}^{2} L^{2} D^{2}+\Delta_{L}^{2} D^{2} B^{2}+\Delta_{D}^{2} B^{2} L^{2}\right)} .
\end{aligned}
$$

Then, the $B$ - $L$ and $D$ asymmetries at the decoupling of the mixing interaction are estimated as 


$$
\begin{aligned}
n_{B-L}\left(T_{\mathrm{dec}}\right) & =\frac{T_{\mathrm{dec}}^{2}}{6}(B-L) \cdot \mu \\
& =\frac{\left(\mu_{B} \Delta_{B}+\mu_{L} \Delta_{L}\right)\left(\Delta_{B}-\Delta_{L}\right) B^{2} L^{2} D^{2}}{\Delta_{B}^{2} L^{2} D^{2}+\Delta_{L}^{2} D^{2} B^{2}+\Delta_{D}^{2} B^{2} L^{2}} \frac{T_{\mathrm{dec}}^{2}}{6}, \\
n_{D}\left(T_{\mathrm{dec}}\right) & =\frac{T_{\mathrm{dec}}^{2} D \cdot \mu}{6} \\
& =\frac{\left(\mu_{B} \Delta_{B}+\mu_{L} \Delta_{L}\right) \Delta_{D} B^{2} L^{2} D^{2}}{\Delta_{B}^{2} L^{2} D^{2}+\Delta_{L}^{2} D^{2} B^{2}+\Delta_{D}^{2} B^{2} L^{2}} \frac{T_{\mathrm{dec}}^{2}}{6}
\end{aligned}
$$

with $T_{\mathrm{dec}}$ being the decoupling temperature of the mixing interaction. From these expressions, it is manifest that $\mu_{B} \Delta_{B}+\mu_{L} \Delta_{L} \neq 0$ is required for the generation of both $B-L$ and $D$ asymmetries. In addition, $\Delta_{B}-\Delta_{L} \neq 0$ and $\Delta_{D} \neq 0$ are necessary for $B-L$ and $D$ asymmetries, respectively. The former condition implies that the mixing interaction must violate $Q_{+}$since the flat direction has $Q_{+}$ charge as a whole and the derivative coupling acts as the chemical potential for $Q_{+}$. The latter conditions reflect the fact that the mixing interaction itself needs to violate $B-L$ and $D$ asymmetries.

Depending on the decoupling temperature, the present asymmetries are estimated as

$$
\begin{aligned}
\frac{n_{B-L}}{s} \simeq & \frac{15}{4 \pi^{2} g_{* s}} \\
& \times \frac{\left(\Delta_{B}-\Delta_{L}\right)\left(\mu_{B}\left(T_{\mathrm{dec}}\right) \Delta_{B}+\mu_{L}\left(T_{\mathrm{dec}}\right) \Delta_{L}\right) B^{2} L^{2} D^{2}}{T_{\mathrm{dec}}\left(\Delta_{B}^{2} L^{2} D^{2}+\Delta_{L}^{2} D^{2} B^{2}+\Delta_{D}^{2} B^{2} L^{2}\right)} \\
& \times \begin{cases}1 & \text { for } T_{\mathrm{dec}}<T_{R} \\
\left(\frac{T_{R}}{T_{\mathrm{dec}}}\right)^{5} & \text { for } T_{\mathrm{dec}}>T_{R}\end{cases}
\end{aligned}
$$$$
\frac{n_{D}}{s} \simeq \frac{15}{4 \pi^{2} g_{* s}} \frac{\Delta_{D}\left(\mu_{B}\left(T_{\mathrm{dec}}\right) \Delta_{B}+\mu_{L}\left(T_{\mathrm{dec}}\right) \Delta_{L}\right) B^{2} L^{2} D^{2}}{T_{\mathrm{dec}}\left(\Delta_{B}^{2} L^{2} D^{2}+\Delta_{L}^{2} D^{2} B^{2}+\Delta_{D}^{2} B^{2} L^{2}\right)}
$$$$
\times \begin{cases}1 & \text { for } T_{\mathrm{dec}}<T_{R}, \\ \left(\frac{T_{R}}{T_{\mathrm{dec}}}\right)^{5} & \text { for } T_{\mathrm{dec}}>T_{R} .\end{cases}
$$

If annihilation process works sufficiently, only asymmetric part of $D$ charges, that is, the $\Psi$ (or $\bar{\Psi}$ ) particle remains, which is responsible for the present dark matter. Since the ratio of the $D$ asymmetry to the $B-L$ one is determined by the amounts of violations of the mixing interaction,

$$
\frac{n_{D} / s}{n_{B-L} / s}=\frac{\Delta_{D}}{\Delta_{B}-\Delta_{L}},
$$

it is fixed after the freeze-out of the mixing interaction. After the decay of the flat direction, the sphaleron process converts a part of the $B-L$ asymmetry into baryon asymmetry so that the present baryon-to-entropy ratio is given by,

$$
\frac{n_{B}}{s}=\frac{8}{23} \frac{n_{B-L}}{s} .
$$

Therefore, the DM mass given by

$$
M_{\Psi} \simeq 1.6 \mathrm{GeV} \times \frac{\Delta_{B}-\Delta_{L}}{\Delta_{D}}
$$

can explain not only the present dark matter abundance,

$$
\frac{\rho_{\mathrm{DM}}}{s}=\frac{M_{\Psi} n_{D}}{s} \simeq 4.1 \times 10^{-10} \mathrm{GeV},
$$

but also the present baryon-to-entropy ratio,

$$
\frac{n_{B}}{s}=(8.1-9.4) \times 10^{-11} \text {. }
$$

\section{The case with both a $B-L-D$ mixing interaction and a $B-L$ violating interaction}

Let us now consider a case with a $B-L$ violating interaction as well as a $B-L-D$ violating (mixing) interaction, which can accommodate a TeV scale dark matter model. Denoting the amounts of the charge violation as $\Delta_{B 1}$ and $\Delta_{L 1}$ for the $B-L$ violating interaction and $\Delta_{B 2}, \Delta_{L 2}$ and $\Delta_{D}$ for the $B-L-D$ mixing interaction, respectively, the $B, L$ and $D$ asymmetries satisfy the following relation:

$$
\begin{aligned}
\sum_{m} \bar{\Xi}_{m} n_{m}= & \Delta_{L 1} \Delta_{D} n_{B}-\Delta_{B 1} \Delta_{D} n_{L} \\
& -\left(\Delta_{B 2} \Delta_{L 1}-\Delta_{B 1} \Delta_{L 2}\right) n_{D}=0,
\end{aligned}
$$

where $\bar{\Xi}_{m}$ is defined as

$$
\begin{aligned}
\bar{\Xi}_{m} \equiv & \Delta_{L 1} \Delta_{D} B_{m}-\Delta_{B 1} \Delta_{D} L_{m} \\
& -\left(\Delta_{B 2} \Delta_{L 1}-\Delta_{B 1} \Delta_{L 2}\right) D_{m},
\end{aligned}
$$

if there are no other symmetry breaking interactions. This relation is rewritten in terms of chemical potential as

$$
\bar{\Xi} \cdot \mu=0 \text {. }
$$

Then, the physical chemical potential of $m$-th field can be expressed as

$$
\mu_{m}=\bar{\mu}_{m}-\beta \bar{\Xi}_{m}
$$

with the numerical constant $\beta$ given by

$$
\beta=\frac{\left(\mu_{B} \Delta_{L 1} B^{2}-\mu_{L} \Delta_{B 1} L^{2}\right) \Delta_{D}}{\Delta_{L 1}^{2} \Delta_{D}^{2} B^{2}+\Delta_{B 1}^{2} \Delta_{D}^{2} L^{2}+\left(\Delta_{B 2} \Delta_{L 1}-\Delta_{B 1} \Delta_{L 2}\right)^{2} D^{2}} .
$$

From this expression, the $B-L$ and $D$ asymmetries at the decoupling of the mixing interaction ${ }^{11}$ are estimated as

\footnotetext{
${ }^{11}$ We assume that the $B-L$ violating interaction decouples after the decoupling of the $B-L-D$ mixing interaction since the symmetry the former breaks is smaller than that the latter does.
} 


$$
\begin{gathered}
n_{B}\left(T_{\operatorname{dec} 2}\right)=\frac{\left[\left(\mu_{B} \Delta_{B 1}+\mu_{L} \Delta_{L 1}\right) \Delta_{B 1} \Delta_{D}^{2} L^{2}+\mu_{B}\left(\Delta_{B 2} \Delta_{L 1}-\Delta_{B 1} \Delta_{L 2}\right)^{2} D^{2}\right] B^{2}}{\Delta_{D}^{2}\left(\Delta_{L 1}^{2} B^{2}+\Delta_{B 1}^{2} L^{2}\right)+\left(\Delta_{B 2} \Delta_{L 1}-\Delta_{B 1} \Delta_{L 2}\right)^{2} D^{2}} \frac{T_{\operatorname{dec} 2}^{2}}{6}, \\
n_{L}\left(T_{\operatorname{dec} 2}\right)=\frac{\left[\left(\mu_{B} \Delta_{B 1}+\mu_{L} \Delta_{L 1}\right) \Delta_{L 1} \Delta_{D}^{2} B^{2}+\mu_{L}\left(\Delta_{B 2} \Delta_{L 1}-\Delta_{B 1} \Delta_{L 2}\right)^{2} D^{2}\right] L^{2}}{\Delta_{D}^{2}\left(\Delta_{L 1}^{2} B^{2}+\Delta_{B 1}^{2} L^{2}\right)+\left(\Delta_{B 2} \Delta_{L 1}-\Delta_{B 1} \Delta_{L 2}\right)^{2} D^{2}} \frac{T_{\operatorname{dec} 2}^{2}}{6}, \\
n_{D}\left(T_{\operatorname{dec} 2}\right)=\frac{\Delta_{D}\left(\mu_{B} \Delta_{L 1} B^{2}-\mu_{L} \Delta_{B 1} L^{2}\right)\left(\Delta_{B 2} \Delta_{L 1}-\Delta_{B 1} \Delta_{L 2}\right) D^{2}}{\left.\Delta_{D}^{2}\left(\Delta_{L 1}^{2} B^{2}+\Delta_{B 1}^{2} L^{2}\right)+\left(\Delta_{B 2} \Delta_{L 1}-\Delta_{B 1} \Delta_{L 2}\right)^{2} D^{2}\right)} \frac{T_{\operatorname{dec} 2}^{2}}{6},
\end{gathered}
$$

with $T_{\operatorname{dec} 2}$ being the decoupling temperature of the mixing interaction. Thus, it is manifest that the following three conditions must be satisfied for successful dark matter genesis (cogenesis):

$$
\begin{aligned}
& \Delta_{D} \neq 0, \quad \Delta_{B 2} \Delta_{L 1}-\Delta_{B 1} \Delta_{L 2} \neq 0, \\
& \mu_{B} \Delta_{L 1} B^{2}-\mu_{L} \Delta_{B 1} L^{2} \neq 0 .
\end{aligned}
$$

The first condition implies that $D$ violation is necessary for the genesis of $D$ asymmetry. The second condition reflects the fact that, only when $\Delta_{B 2} \Delta_{L 1}-\Delta_{B 1} \Delta_{L 2} \neq 0$, $\bar{\Xi}_{m}$ depends on $D_{m}$ and the effective chemical potential of $D$ charged particles arises. The last condition implies that the ratio of $\mu_{B} B^{2}$ to $\mu_{L} L^{2}$ should not be equal to the ratio of $\Delta_{B 1}$ to $\Delta_{L 1}$. The former represents the ratio of the would-be baryon and lepton asymmetries generated by the effective chemical potential $\bar{\mu}_{m}$ without the constraint (65). Note also that, if both of the ratios coincide, the constant $\beta$ given in Eq. (69) vanishes. Thus, the last condition requires that the $B-L-D$ mixing interaction as well as the $B-L$ violating interaction must really work to generate $B$ and $L$ asymmetries, which involves the generation of $D$ asymmetry.

Depending on the decoupling temperature, the present $D$ asymmetry is estimated in terms of number-to-entropy ratio as

$$
\frac{n_{D}}{s} \simeq \frac{15}{4 \pi^{2} g_{* s}} \frac{\Delta_{D}\left[\mu_{B}\left(T_{\operatorname{dec} 2}\right) \Delta_{L 1} B^{2}-\mu_{L}\left(T_{\mathrm{dec} 2}\right) \Delta_{B 1} L^{2}\right]\left(\Delta_{B 2} \Delta_{L 1}-\Delta_{B 1} \Delta_{L 2}\right) D^{2}}{\left[\Delta_{D}^{2}\left(\Delta_{L 1}^{2} B^{2}+\Delta_{B 1}^{2} L^{2}\right)+\left(\Delta_{B 2} \Delta_{L 1}-\Delta_{B 1} \Delta_{L 2}\right)^{2} D^{2}\right] T_{\operatorname{dec} 2}} \times \begin{cases}1 & \text { for } T_{\operatorname{dec} 2}<T_{R}, \\ \left(\frac{T_{R}}{T_{\operatorname{dec} 2}}\right)^{5} & \text { for } T_{\operatorname{dec} 2}>T_{R} .\end{cases}
$$

On the other hand, the $B-L$ violating interaction continues to generate $B$ and $L$ asymmetries. Since the amounts of variation of these asymmetries generated after the decoupling of the mixing interaction are determined only by the violating interaction, $B$ and $L$ asymmetries must satisfy the following relation:

$$
\begin{aligned}
\frac{n_{B}(T)-\left(a\left(T_{\operatorname{dec} 2}\right) / a(T)\right)^{3} n_{B}\left(T_{\operatorname{dec} 2}\right)}{n_{L}(T)-\left(a\left(T_{\operatorname{dec} 2}\right) / a(T)\right)^{3} n_{L}\left(T_{\operatorname{dec} 2}\right)} & =\frac{\Delta_{B 1}}{\Delta_{L 1} \Leftrightarrow \Delta_{L 1} n_{B}(T)-\Delta_{B 1} n_{L}(T)} \\
& =\left(\frac{a\left(T_{\operatorname{dec} 2}\right)}{a(T)}\right)^{3}\left(\Delta_{L 1} n_{B}\left(T_{\operatorname{dec} 2}\right)-\Delta_{B 1} n_{L}\left(T_{\operatorname{dec} 2}\right)\right) \\
& =\left(\frac{a\left(T_{\operatorname{dec} 2}\right)}{a(T)}\right)^{3} \frac{\left(\mu_{B} \Delta_{L 1} B^{2}-\mu_{L} \Delta_{B 1} L^{2}\right)\left(\Delta_{B 2} \Delta_{L 1}-\Delta_{B 1} \Delta_{L 2}\right)^{2} D^{2}}{\left[\Delta_{D}^{2}\left(\Delta_{L 1}^{2} B^{2}+\Delta_{B 1}^{2} L^{2}\right)+\left(\Delta_{B 2} \Delta_{L 1}-\Delta_{B 1} \Delta_{L 2}\right)^{2} D^{2}\right]} \frac{T_{\operatorname{dec} 2}^{2}}{6} \\
& \equiv C\left(T, T_{\operatorname{dec} 2}\right) .
\end{aligned}
$$

This relation is rewritten in terms of the chemical potential as

$$
\tilde{\Xi} \cdot \mu=\frac{6}{T^{2}} C\left(T, T_{\operatorname{dec} 2}\right),
$$

with

$$
\tilde{\Xi}_{m} \equiv \Delta_{L 1} B_{m}-\Delta_{B 1} L_{m} .
$$

Then, the chemical potential of $m$-th field can be expressed as

$$
\mu_{m}=\bar{\mu}_{m}-\tilde{\beta} \tilde{\Xi}_{m}
$$

with the numerical constant $\tilde{\beta}$ given by 


$$
\tilde{\beta}=\frac{\left(\mu_{B} \Delta_{L 1} B^{2}-\mu_{L} \Delta_{B 1} L^{2}\right)-6 C\left(T, T_{\operatorname{dec} 2}\right) / T^{2}}{\Delta_{L 1}^{2} B^{2}+\Delta_{B 1}^{2} L^{2}} .
$$

The $B-L$ asymmetry at the decoupling of the $B-L$ violating interaction, $T=T_{\mathrm{dec} 1}$, is estimated as

$$
n_{B-L}\left(T_{\operatorname{dec} 1}\right)=\left(\Delta_{B 1}-\Delta_{L 1}\right)\left(\mu_{B} \Delta_{B 1}+\mu_{L} \Delta_{L 1}\right) \frac{B^{2} L^{2}}{B^{2} \Delta_{L 1}^{2}+L^{2} \Delta_{B 1}^{2}} \frac{T_{\operatorname{dec} 1}^{2}}{6}+\frac{\Delta_{L 1} B^{2}+\Delta_{B 1} L^{2}}{\Delta_{L 1}^{2} B^{2}+\Delta_{B 1}^{2} L^{2}} C\left(T_{\operatorname{dec} 1}, T_{\operatorname{dec} 2}\right) .
$$

In the case of $C\left(T_{\operatorname{dec} 1}, T_{\operatorname{dec} 2}\right)=0,{ }^{12}$ depending on the decoupling temperature, the present $B-L$ number-to-entropy ratio is evaluated as

$$
\frac{n_{B-L}}{s}=\frac{15}{4 \pi^{2} g_{* 2}} \frac{\left(\Delta_{B 1}-\Delta_{L 1}\right)\left(\mu_{B}\left(T_{\operatorname{dec} 1}\right) \Delta_{B 1}+\mu_{L}\left(T_{\operatorname{dec} 1}\right) \Delta_{L 1}\right) B^{2} L^{2}}{\left(B^{2} \Delta_{L 1}^{2}+L^{2} \Delta_{B 1}^{2}\right) T_{\operatorname{dec} 1}} \times \begin{cases}1 & \text { for } T_{\operatorname{dec} 1}<T_{R}, \\ \left(\frac{T_{R}}{T_{\operatorname{dec} 1}}\right)^{5} & \text { for } T_{\operatorname{dec} 1}>T_{R} .\end{cases}
$$

After the decay of the flat direction, the sphaleron process switches on and reconfigures the baryon asymmetry so that the present baryon asymmetry is estimated as

$$
\frac{n_{B}}{s}=\frac{30}{23 \pi^{2} g_{* 2}} \frac{\left(\Delta_{B 1}-\Delta_{L 1}\right)\left(\mu_{B}\left(T_{\mathrm{dec} 1}\right) \Delta_{B 1}+\mu_{L}\left(T_{\mathrm{dec} 1}\right) \Delta_{L 1}\right) B^{2} L^{2}}{\left(B^{2} \Delta_{L 1}^{2}+L^{2} \Delta_{B 1}^{2}\right) T_{\mathrm{dec} 1}} \times \begin{cases}1 & \text { for } T_{\mathrm{dec} 1}<T_{R}, \\ \left(\frac{T_{R}}{T_{\mathrm{dec} 1}}\right)^{5} & \text { for } T_{\mathrm{dec} 1}>T_{R} .\end{cases}
$$

The ratio of the $D$ asymmetry to the $B$ - $L$ asymmetry is suppressed by a factor of $\mathcal{O}\left(\left(T_{\operatorname{dec} 1} / T_{\operatorname{dec} 2}\right)^{6}\right)$ apart from a numerical factor, if $T_{R}<T_{\operatorname{dec} 1}, T_{\operatorname{dec} 2}$ and the temperature dependence of the chemical potential is neglected. Therefore, the mass of $\Psi$ given by

$$
M_{\Psi} \sim 1 \mathrm{GeV} \times\left(\frac{T_{\operatorname{dec} 2}}{T_{\operatorname{dec} 1}}\right)^{6}
$$

can explain the present dark matter abundance (63) and the baryon-to-entropy ratio (64) simultaneously. Thus, this scenario can accommodate dark matter with weak scale mass if $T_{\operatorname{dec} 2} / T_{\operatorname{dec} 1} \sim \mathcal{O}\left(10^{1 / 2}\right)$.

One may wonder whether the baryon and dark matter isocurvature perturbation would be too large to be consistent with the present observations. The isocurvature perturbations roughly can be estimated by

$$
\frac{\delta n_{B(D)}}{n_{B(D)}} \simeq \frac{\delta \dot{a}_{+}}{\dot{a}_{+}} \simeq \frac{\delta a_{+}}{a_{+}} \simeq \frac{1}{2 \pi}\left(\frac{H_{\mathrm{inf}}}{M_{*}}\right)^{(n-3 / n-2)},
$$

with $H_{\text {inf }}$ being the Hubble parameter during inflation. Therefore, for large $M_{*}$ compared to the Hubble parameter during inflation, the baryon and dark matter isocurvature perturbations are significantly suppressed.

\section{APPLICATION}

Here we consider concrete examples of spontaneous cogenesis in a flat direction of the MSSM with $B-L=0$ and $B+L \neq 0$. The $\mathrm{AD}$ mechanism works only for a flat

\footnotetext{
${ }^{12}$ This is an appropriate approximation since the asymmetry $\Delta_{L 1} n_{B}-\Delta_{B 1} n_{L}$ at the decoupling of the mixing interaction is considerably suppressed by a factor of $\left(a\left(T_{\operatorname{dec} 2}\right) / a(T)\right)^{3}$. Even if $C\left(T, T_{\operatorname{dec} 2}\right)$ has a non-negligible value, the present asymmetry is easily calculated by the same procedure.
}

direction with $B-L$ charge, otherwise the sphaleron process would wash out $B$ and $L$ asymmetries before the electroweak phase transition. ${ }^{13}$ On the other hand, spontaneous baryo/cogenesis can work even for a flat direction without $B-L$ charge. Therefore, in order to avoid too large asymmetry generated by the AD mechanism, we investigate the cogenesis scenario along a flat direction without $B-L$ charge.

For definiteness, we choose the $Q Q Q L$ flat direction parameterized as

$$
\begin{array}{ll}
Q_{1}^{r}=\frac{1}{\sqrt{2}}\left(\begin{array}{c}
f e^{i \alpha_{B} / 3} \\
0
\end{array}\right), & Q_{2}^{b}=\frac{1}{\sqrt{2}}\left(\begin{array}{c}
0 \\
f e^{i \alpha_{B} / 3}
\end{array}\right), \\
Q_{3}^{g}=\frac{1}{\sqrt{2}}\left(\begin{array}{c}
f e^{i \alpha_{B} / 3} \\
0
\end{array}\right), & L_{1}=\frac{1}{\sqrt{2}}\left(\begin{array}{c}
0 \\
f e^{i \alpha_{L}}
\end{array}\right),
\end{array}
$$

where $r, b$ and $g$ are color indices, and $f, \alpha_{B}$ and $\alpha_{L}$ are real parameters. In this example, all $f_{i}$ 's defined in Eq. (9) are identical and denoted by $f$, which yields $\phi=f$ for the representative scalar field. Since the weak gauge bosons become massive due to the large VEV of the flat direction, the sphaleron process does not work until the decay of this flat direction. For this flat direction, $\xi, Q_{i}^{ \pm}$and $v_{a}$ defined in Eqs. (10)-(12) and (18), are given by

\footnotetext{
${ }^{13}$ If $Q$ balls are formed and do not evaporate away before the electroweak phase transition, the AD mechanism is still viable even for a flat direction with vanishing $B-L$ charge. Since $Q$ balls protect the asymmetries from the sphaleron process, both baryon and lepton asymmetries can be generated in the Universe. However, in our concrete models, $Q$ balls are not formed because the flat direction quickly decays just after it starts the oscillation, as discussed later.
} 


$$
\begin{aligned}
\tan \xi & =1, \quad Q_{i}^{+}=\frac{B_{i}+L_{i}}{\sqrt{2}}, \\
Q_{i}^{-} & =\frac{-B_{i}+L_{i}}{\sqrt{2}}, \quad v_{a}=\frac{\phi}{\sqrt{2}} .
\end{aligned}
$$

Since some of the fields become massive due to the direct coupling to the flat direction when it acquires a large VEV, the quantities that represent the light degrees of freedom of baryon, lepton, and dark matter are estimated as

$$
B^{2} \equiv \sum_{m} \kappa_{m} g_{m} B_{m}^{2}=\frac{23}{2}, \quad L^{2}=\frac{81}{2}, \quad D^{2}=12,
$$

respectively. The reason why half-integer baryon and lepton number squared appear comes from the fact that some of massless eigenstates are realized as mixed states of (s) quarks and (s)leptons.

In order to evaluate the effective chemical potential, we discuss the evolution of the flat direction. Though the scalar potential along the flat direction vanishes in the supersymmetric and renormalizable limit, it is lifted up by a nonrenormalizable superpotential,

$$
W_{\mathrm{NR}}=\frac{Q Q Q L}{4 M_{*}},
$$

with $M_{*}$ being a cutoff scale for this interaction and SUSYbreaking effect. Assuming the negative Hubble induced mass squared, the flat direction is destabilized at the origin and acquires a large VEV. After inflation, the (negative) thermal logarithmic potential Eq. (30) arises from thermal plasma that exists even in the inflaton dominated era as a subdominant component of the Universe. Note that all the gauge fields become massive since the $Q Q Q L$ flat direction completely breaks the SM gauge groups. Thus, thermal logarithmic potential coming from the two-loop finite temperature effects is dominated by the effect of the running of the top Yukawa coupling with the nonzero field value of the scalar fields. Since the one-loop correction to the top Yukawa coupling is negative, the thermal logarithmic correction turns out to be negative [23]. Then, the dynamics of the $Q Q Q L$ flat direction follows the discussion in Sec. II A with $n=4$. During the inflaton oscillation dominated era, this potential has a minimum at

$$
\phi_{\min } \sim \begin{cases}\left(H M_{*}\right)^{1 / 2} & \text { for } T>T_{c}, \\ \alpha^{1 / 6}\left(T^{2} M_{*}\right)^{1 / 3} & \text { for } T<T_{c},\end{cases}
$$

with the critical temperature $T_{c}$ given by

$$
T_{c} \equiv \alpha^{1 / 8} T_{R}^{3 / 4} M_{G}^{3 / 8} M_{*}^{-1 / 8} .
$$

After reheating, the negative Hubble induced mass squared vanishes. Then, the potential minimum is determined by the balance of the thermal logarithmic potential and the $F$ term, and is given by

$$
\phi_{\min } \sim \alpha^{1 / 6}\left(T^{2} M_{*}\right)^{1 / 3} .
$$

The flat direction follows this minimum until the mass term ${ }^{14}$ overwhelms the thermal logarithmic potential at

$$
T \sim \alpha^{-1 / 4} m_{3 / 2}^{3 / 4} M_{*}^{1 / 4}, \quad \phi \sim\left(m_{3 / 2} M_{*}\right)^{1 / 2} .
$$

Below this temperature, the flat direction starts its oscillation around the origin and decays quickly since its decay rate $\Gamma \sim m_{3 / 2} / 8 \pi$ is much larger than the Hubble parameter $H \sim T^{2} / M_{G} \sim \alpha^{-1 / 2} m_{3 / 2}\left(f / M_{G}\right)$. Thus, $Q$ balls would not be formed because of the quick decay of the flat direction.

While the NG boson $a_{+}$slow-rolls, its velocity is estimated from Eqs. (36), (89), and (91) as

$$
\begin{aligned}
\left|\dot{a}_{+}\right| & =\frac{Q^{+}}{H v_{a}} M_{A}^{4}=\frac{a_{3 / 2} m_{3 / 2}}{8 M_{*} H} \phi_{\min }^{3} \\
& \simeq \begin{array}{ll}
\frac{a_{3 / 2}}{8} m_{3 / 2} \phi_{\min } & \text { for } T>T_{c}, T_{R}, \\
\frac{\alpha^{1 / 3} a_{3 / 2} m_{3 / 2} M_{G} T_{R}^{2}}{8 M_{*}^{1 / 3} T^{8 / 3}} \phi_{\min } & \text { for } T_{R}<T<T_{c}, \\
\frac{\alpha^{1 / 3} a_{3 / 2} m_{3 / 2} M_{G}}{8 M_{*}^{1 / 3} T^{2 / 3}} \phi_{\min } & \text { for } T<T_{R} .
\end{array}
\end{aligned}
$$

Then, the chemical potential $\bar{\mu}_{m}$ defined in Eq. (25) is given by

$$
\bar{\mu}_{m}=-\frac{B_{m}+L_{m}}{\sqrt{2} v_{a}} \dot{a}_{+}=B_{m} \mu_{B}+L_{m} \mu_{L}
$$

$\left|\mu_{B}\right|=\left|\mu_{L}\right| \simeq \frac{a_{3 / 2}}{8} m_{3 / 2} \times \begin{cases}1 & \text { for } T>T_{c}, T_{R}, \\ \frac{\alpha^{1 / 3} M_{G} T_{R}^{2}}{M_{*}^{1 / 3} T^{8 / 3}} & \text { for } T_{R}<T<T_{c}, \\ \frac{\alpha^{1 / 3} M_{G}}{M_{*}^{1 / 3} T^{2 / 3}} & \text { for } T<T_{R},\end{cases}$

where we have used Eqs. (24) and (93). The slow-roll violating condition of the NG boson given in Eq. (32) is expressed in terms of the temperature as

$$
T<T_{\text {slow }} \equiv \begin{cases}\left(a_{3 / 2} m_{3 / 2} M_{G} T_{R}^{2}\right)^{1 / 4} & \text { for } T_{\text {slow }}>T_{c}, T_{R}, \\ \left(\frac{\alpha a_{3 / 2}^{3} m_{3 / 2}^{3} M_{G}^{6} T_{R}^{12}}{M_{*}}\right)^{1 / 20} & \text { for } T_{c}>T_{\text {slow }}>T_{R}, \\ \left(\frac{\alpha a_{3 / 2}^{3} m_{3 / 2}^{3} M_{G}^{6}}{M_{*}}\right)^{1 / 8} & \text { for } T_{R}>T_{\text {slow }} .\end{cases}
$$

Equation (95) is valid for $T>T_{\text {slow }}$. Otherwise, they are severely suppressed because the field $a_{+}$oscillates rapidly [20].

\footnotetext{
${ }^{14}$ Here we assume the gravity mediated SUSY-breaking mechanism.
} 


\section{A. The case with only a $B-L-D$ mixing interaction}

First we consider a spontaneous cogenesis scenario with only a $B-L-D$ mixing interaction discussed in Sec. II B 1 . As an illustrative example, we consider the following superpotential:

$$
W_{\text {mix }}=\frac{\bar{u} \bar{d} \bar{d} \Psi^{2}}{\Lambda^{2}},
$$

which induces a $B-L-D$ mixing interaction,

$$
\mathcal{L}_{\text {vio }}=\frac{1}{\Lambda^{2}} \tilde{\bar{u}}_{R} \tilde{\bar{d}}_{R} \bar{d}_{R} \Psi^{2}+\text { H.c., }
$$

where the tilde represents the fermion component of a chiral multiplet. Here we impose $Z_{2}$ symmetry on the DM sector. Some flavor and color combinations of $\bar{u} \bar{d} \bar{d}$ (e.g., $\bar{u}_{R 1}^{b} \bar{d}_{R 2}^{g} \bar{d}_{R 3}^{r}$ ) remain massless even though the $Q Q Q L$ flat direction acquires a large VEV. Then, this mixing interaction is in thermal equilibrium at high temperature. More specifically, the rate of the interaction is given by $\Gamma \sim T^{5} /\left(8 \pi \Lambda^{4}\right)$, which leads to its decoupling temperature,

$$
T_{\operatorname{dec} 1}= \begin{cases}8 \pi \frac{\Lambda^{4}}{M_{G} T_{R}^{2}} & \text { for } T_{\operatorname{dec} 1}>T_{R}, \\ (8 \pi)^{1 / 3} \frac{\Lambda^{4 / 3}}{M_{G}^{1 / 3}} & \text { for } T_{\operatorname{dec} 1}<T_{R} .\end{cases}
$$

Since the amounts of $B, L$ and $D$ violation in this interaction are given by

$$
\Delta_{B}=-1, \quad \Delta_{L}=0, \quad \Delta_{D}=2,
$$

the operators $\Xi_{m}^{(1)}$ and $\Xi_{m}^{(2)}$ in Eq. (51) are written as

$$
\Xi_{m}^{(1)}=-2 L_{m}, \quad \Xi_{m}^{(2)}=-D_{m}-2 B_{m} .
$$

Then, the present $B-L$ and $D$ asymmetries are estimated from Eqs. (58), (59), (94), and (95), as

$$
\begin{aligned}
\frac{n_{B-L}}{s}= & \frac{1035}{928 \pi^{2} g_{* s}} \frac{a_{3 / 2} m_{3 / 2}}{T_{\mathrm{dec}}} \\
& \times \begin{cases}\left(\frac{T_{R}}{T_{\mathrm{dec}}}\right)^{5} & \text { for } T_{\mathrm{dec}}>T_{c}, T_{R}, \\
\frac{\alpha^{1 / 3} M_{G} T_{R}^{2}}{M_{*}^{1 / 3} T_{\mathrm{dec}}^{8 / 3}}\left(\frac{T_{R}}{T_{\mathrm{dec}}}\right)^{5} & \text { for } T_{R}<T_{\mathrm{dec}}<T_{c}, \\
\frac{\alpha^{1 / 3} M_{G}}{M_{*}^{1 / 3} T_{\mathrm{dec}}^{2 / 3}} & \text { for } T_{\mathrm{dec} 2}<T_{R},\end{cases} \\
\frac{\left|n_{D}\right|}{s}= & \frac{1035}{464 \pi^{2} g_{* s}} \frac{a_{3 / 2} m_{3 / 2}}{T_{\mathrm{dec}}} \\
& \times \begin{cases}\left(\frac{T_{R}}{T_{\mathrm{dec}}}\right)^{5} & \text { for } T_{\mathrm{dec}}>T_{c}, T_{R}, \\
\frac{\alpha^{1 / 3} M_{G} T_{R}^{2}}{M_{*}^{1 / 3} T_{\mathrm{dec}}^{8 / 3}}\left(\frac{T_{R}}{T_{\mathrm{dec}}}\right)^{5} & \text { for } T_{R}<T_{\mathrm{dec}}<T_{c}, \\
\frac{\alpha^{1 / 3} M_{G}}{M_{*}^{1 / 3} T_{\mathrm{dec}}^{2 / 3}} & \text { for } T_{\mathrm{dec}}<T_{R} .\end{cases}
\end{aligned}
$$

Here we have assumed that $\dot{a}_{+}<0$.
A part of the $B-L$ asymmetry is transferred to $B$ asymmetry through the sphaleron process that works only after the $Q Q Q L$ flat direction decay. The present baryon asymmetry of the Universe is, then, estimated as

$$
\begin{aligned}
\frac{n_{B}}{s}= & \frac{8}{23} \frac{n_{B-L}}{s} \simeq \frac{45}{116 \pi^{2} g_{* s}} \frac{m_{3 / 2}}{T_{\mathrm{dec}}} \\
& \times \begin{cases}\left(\frac{T_{R}}{T_{\mathrm{dec}}}\right)^{5} & \text { for } T_{\mathrm{dec}}>T_{c}, T_{R}, \\
\frac{\alpha^{1 / 3} M_{G} T_{R}^{2}}{M_{*}^{1 / 3} T_{\mathrm{dec}}^{8 / 3}}\left(\frac{T_{R}}{T_{\mathrm{dec}}}\right)^{5} & \text { for } T_{R}<T_{\mathrm{dec}}<T_{c}, \\
\frac{\alpha^{1 / 3} M_{G}}{M_{*}^{1 / 3} T_{\mathrm{dec}}^{2 / 3}} & \text { for } T_{\mathrm{dec}}<T_{R} .\end{cases}
\end{aligned}
$$

The observed baryon asymmetry given in Eq. (64) can be explained with the parameters, for example, $T_{R} \simeq$ $T_{\mathrm{dec}} \simeq 10^{12} \mathrm{GeV}, m_{3 / 2} \simeq 4.5 \times 10^{5} \mathrm{GeV}$, and $M_{*}>2 \times$ $10^{27} \mathrm{GeV}$. Notice that the conditions $T_{R}>T_{c}, T_{\text {slow }}$ are satisfied for these parameters. If annihilation of the $\Psi$ fields occurs sufficiently (this issue is discussed later), the $\bar{\Psi}$ field with the mass of

$$
M_{\Psi} \simeq 0.8 \mathrm{GeV}
$$

is also responsible for the present dark matter abundance given in Eq. (63).

One may wonder such a high reheating temperature generates too many lightest supersymmetric particles (LSPs) such as neutralinos that could overclose the Universe or induces the gravitino problem. These problems can be avoided if the mass of LSP is small enough, as is the case with the axino LSP scenario. Such a small LSP mass is a natural assumption since a light particle is required for the annihilation of DMs in the first place.

\section{B. The case with both a $B-L-D$ mixing interaction and a $B-L$ violating interaction}

We consider a scenario with a $B-L$ violating operator given by

$$
\mathcal{L}_{\text {vio }}=\frac{1}{\Lambda^{\prime 2}} \bar{d}_{R 3}^{3} \tilde{e}_{L 2} \tilde{\nu}_{L 3}+\text { H.c., }
$$

as well as the $B-L-D$ mixing interaction given in Eq. (97). The latter operator comes from a nonrenormalizable superpotential,

$$
W_{\text {vio }}=\frac{\bar{d}_{3}^{3} L_{2} L_{3}}{\Lambda^{\prime 2}},
$$

with $\Lambda^{\prime}$ being a cutoff scale. As discussed in the previous subsection, by taking adequate flavors and colors, all the fields constituting of this interaction remain massless even though the $Q Q Q L$ flat direction acquires a large VEV. The amounts of $B, L$ and $D$ violation of these interactions are given by 


$$
\begin{array}{ll}
\Delta_{B 1}=-1, & \Delta_{L 1}=2, \\
\Delta_{B 2}=-1, & \Delta_{L 2}=0, \quad \Delta_{D}=2,
\end{array}
$$

which leads to the operators $\bar{\Xi}_{m}$ [Eq. (66)] and $\tilde{\Xi}_{m}$ [Eq. (77)], respectively,

$$
\bar{\Xi}_{m}=4 B_{m}+2 L_{m}+2 D_{m}, \quad \tilde{\Xi}_{m}=2 B_{m}+L_{m} .
$$

As discussed in Sec. II B 2, D asymmetry is generated thanks to the charge mixing/symmetry breaking interactions. Depending on the decoupling temperature, from Eqs. (74), (94), and (95), the present $D$ asymmetry is estimated as

$$
\begin{aligned}
\frac{n_{D}}{s}= & \frac{5715}{1576 \pi^{2} g_{* s}} \frac{a_{3 / 2} m_{3 / 2}}{T_{\operatorname{dec} 2}} \\
& \times \begin{cases}\left(\frac{T_{R}}{T_{\operatorname{dec} 2} 2}\right)^{5} & \text { for } T_{\operatorname{dec} 2}>T_{c}, T_{R}, \\
\frac{\alpha^{1 / 3} M_{G} T_{R}^{2}}{M_{*}^{1 / 3} T_{\operatorname{dec} 2}^{8 / 3}}\left(\frac{T_{R}}{T_{\operatorname{dec} 2}}\right)^{5} & \text { for } T_{R}<T_{\operatorname{dec} 2}<T_{c}, \\
\frac{\alpha^{1 / 3} M_{G}}{M_{*}^{1 / 3} T_{\operatorname{dec} 2}^{2 / 3}} & \text { for } T_{\operatorname{dec} 2}<T_{R},\end{cases}
\end{aligned}
$$

where we have assumed $\dot{a}_{+}>0$.

Assuming $T_{\operatorname{dec} 1}<T_{\operatorname{dec} 2}$, the factor $C\left(T, T_{\operatorname{dec} 2}\right)$ given in Eq. (75) is evaluated as

$$
C\left(T, T_{\operatorname{dec} 2}\right)=\left(\frac{a\left(T_{\operatorname{dec} 2}\right)}{a(T)}\right)^{3} \frac{254 \mu_{B}\left(T_{\operatorname{dec} 2}\right) T_{\operatorname{dec} 2}^{2}}{197},
$$

with $\mu_{B}(T)=\mu_{L}(T)$. Then, the resultant $B-L$ asymmetry at the decoupling temperature $T_{\operatorname{dec} 1}$ is given by

$$
\begin{aligned}
n_{B-L}\left(T_{\operatorname{dec} 1}\right)= & -\frac{1863 \mu_{B}\left(T_{\operatorname{dec} 1}\right)}{692} T_{\operatorname{dec} 1}^{2} \\
& -\frac{35}{173} C\left(T_{\operatorname{dec} 1}, T_{\operatorname{dec} 2}\right) .
\end{aligned}
$$

Since $a^{3} \mu(T) T^{2}$ increases rapidly as $T$ decreases, the second term in Eq. (112) is negligible. Then, depending on the decoupling temperature, from Eqs. (82) and (95), the present $B$ asymmetry is estimated as

$$
\begin{aligned}
\frac{n_{B}}{s} \simeq & \frac{3645}{1384 \pi g_{* s}} \frac{a_{3 / 2} m_{3 / 2}}{T_{\operatorname{dec} 1}} \\
& \times \begin{cases}\left(\frac{T_{R}}{T_{\operatorname{dec} 1}}\right)^{5} & \text { for } T_{\operatorname{dec} 1}>T_{c}, T_{R}, \\
\frac{\alpha^{1 / 3} M_{G} T_{R}^{2}}{M_{*}^{1 / 3} T_{\operatorname{dec} 1}^{8 / 3}}\left(\frac{T_{R}}{T_{\operatorname{dec} 1}}\right)^{5} & \text { for } T_{R}<T_{\operatorname{dec} 1}<T_{c}, \\
\frac{\alpha^{1 / 3} M_{G}}{M_{*}^{1 / 3} T_{\operatorname{dec} 1}^{2 / 3}} & \text { for } T_{\operatorname{dec} 1}<T_{R} .\end{cases}
\end{aligned}
$$

Here we have taken into account the sphaleron process that reconfigures $B$ and $L$ asymmetries after the decay of the $Q Q Q L$ flat direction. The observed baryon asymmetry [Eq. (64)] and the dark matter abundance (Eq. (63)) can be explained simultaneously, for example, by the DM mass given by

$$
m_{\Psi} \simeq 1.6 \mathrm{TeV},
$$

with the parameters, $T_{R} \simeq T_{\mathrm{dec} 1} \simeq 10^{12} \mathrm{GeV}, m_{3 / 2} \simeq$ $10^{5} \mathrm{GeV}, M_{*}>5 \times 10^{27} \mathrm{GeV}$, and $T_{\operatorname{dec} 2} \simeq 3 \times 10^{12} \mathrm{GeV}$, if the annihilation process works sufficiently.

Note that the condition $T_{\operatorname{dec} 1} \lesssim T_{\operatorname{dec} 2}$ is a natural assumption because they are both dimension 6 operators and hence their cutoff scales have similar magnitudes with $\Lambda^{\prime} \lesssim \Lambda$. Operators whose dimension is less than 6 would decouple at higher temperature in the inflaton oscillation dominated era and at lower temperature in the radiation dominated era. Thus, such interactions may never be in thermal equilibrium in the cosmic history and hence we do not consider them.

\section{Dark matter annihilation}

In order for a cogenesis scenario to be successful, the symmetric part of DM must be annihilated enough at thermal freeze-out. Such an annihilation process works significantly when its annihilation cross section satisfies $\left\langle\sigma v_{\text {rel }}\right\rangle>10^{-9} \mathrm{GeV}^{-2}[4,24]$. Here we show that this issue is accomplished by embedding our scenario in the NMSSM, which can explain the origin of the dark matter mass as well. Instead of giving the mass term of dark matter $W_{\text {mass }}=M_{\Psi} \Psi \bar{\Psi}$ and the $\mu$ term $W=\mu H_{u} H_{d}$ explicitly, we consider the following superpotential:

$$
\Delta W=\lambda_{\Psi} S \Psi \bar{\Psi}+\lambda_{H} S H_{u} H_{d}+\frac{\kappa}{3} S^{3},
$$

where $S$ is an additional singlet field and $\lambda_{\Psi}, \lambda_{H}$ and $\kappa$ are numerical coefficients. Induced by the soft SUSY-breaking effect, $S$ acquires a nonvanishing VEV, $\langle S\rangle$, which generates the mass term of dark matter and the $\mu$ term,

$$
M_{\Psi}=\lambda_{\Psi}\langle S\rangle, \quad \mu=\lambda_{H}\langle S\rangle .
$$

For $\lambda_{\Psi} \simeq \lambda_{H} \simeq \mathcal{O}(1)$ and $\langle S\rangle \simeq 10^{2-3} \mathrm{GeV}$, we can obtain both the dark matter and the Higgs masses with the electroweak scale. By decomposing the field $S$ into the radial and the phase components $S=(s / \sqrt{2}) e^{i a / s}$ with $s$ replaced by the VEV, $s=\sqrt{2}\langle S\rangle, \Psi$ and $\bar{\Psi}$ can annihilate into the pair of $a$ field if its mass is smaller than $M_{\Psi} \cdot{ }^{15}$ This annihilation process occurs through the following interaction:

$$
\Delta \mathcal{L}=M_{\Psi} \Psi \bar{\Psi} e^{i a / s} \ni-\frac{M_{\Psi}}{s^{2}} \Psi \bar{\Psi} a^{2},
$$

with the annihilation cross section given by

$$
\left\langle\sigma v_{\mathrm{rel}}\right\rangle=\frac{1}{16 \pi} \frac{M_{\Psi}^{2}}{s^{4}}=\frac{\lambda_{H}^{4} M_{\Psi}^{2}}{64 \pi \mu^{4}} .
$$

\footnotetext{
${ }^{15}$ The field $a$ acquires the mass from the $A$ term. Here we assume that it is smaller than the soft mass $\simeq m_{3 / 2}$.
} 
For $M_{\Psi} \simeq s \simeq 1 \mathrm{TeV}$, we obtain

$$
\left\langle\sigma v_{\text {rel }}\right\rangle \sim 10^{-8} \mathrm{GeV}^{-2},
$$

which is large enough to annihilate the symmetric component of dark matter. For $M_{\Psi} \simeq 1 \mathrm{GeV}$, the annihilation cross section seems too small to annihilate the symmetric part of dark matter significantly. This problem can be avoided if we introduce a bare $\mu$ term,

$$
\Delta W_{H}=\mu_{0} H_{u} H_{d},
$$

in addition to the superpotential (115). In this case, the observed $\mu$ term is expressed as

$$
\mu=\lambda_{H}\langle S\rangle+\mu_{0} .
$$

Then, the annihilation cross section (118) is enhanced by a factor of $\left(\mu /\left(\mu-\mu_{0}\right)\right)^{4}$ and it can be large enough to annihilate the symmetric part of DM sufficiently.

\section{CONCLUSION AND DISCUSSION}

In this paper, we have discussed an ADM model as an extension of the spontaneous baryogenesis in the supersymmetric standard model. A slow-rolling scalar field derivatively coupled to the matter current is identified with a flat direction in the supersymmetric standard model, which arises as a consequence of the Nambu-Goldstone's theorem. In this setup, we have explicitly shown that the present abundances of the baryon and the dark matter can be naturally explained by introducing a nonrenormalizable $B-L-D$ mixing interaction and a few $\mathrm{GeV}$ dark matter mass. Moreover, in the case that there is a $B-L$ violating operator in addition to such a mixing interaction, the baryon asymmetry can be slightly enhanced, which enables us to accommodate dark matter with a TeV scale mass. On the other hand, such an enhancement of the baryon asymmetry does not happen for a slow-rolling scalar field derivatively coupled the dark matter current.

We have found that our model favors relatively high reheating temperature and heavy gravitino mass, which may require some constraints on the mass spectrum of SUSY particles or introducing another new physical degree of freedom. However, our model can be naturally embedded in the NMSSM, which offers us a clue to solve this problem as well as the annihilation mode for the symmetric part of dark matter. It would be interesting to investigate its structure to satisfy the requirements of our scenario in more detail.

\section{ACKNOWLEDGMENTS}

We would like to thank W. Buchmüller, K. Sakurai, T. Suyama, and F. Takahashi for helpful comments and discussions. This work is supported in part by JSPS Grant-inAid for Scientific Research No. 21740187 (M. Y.).
[1] E. Komatsu et al. (WMAP Collaboration), Astrophys. J. Suppl. Ser. 192, 18 (2011).

[2] For review, see G. Bertone, D. Hooper, and J. Silk, Phys. Rep. 405, 279 (2005).

[3] A. D. Sakharov, Pis'ma Zh. Eksp. Teor. Fiz. 5, 32 (1967) [JETP Lett. 5, 24 (1967)].

[4] E.W. Kolb and M.S. Turner, The Early Universe (Westview Press, Boulder, CO, 1990).

[5] I. Affleck and M. Dine, Nucl. Phys. B249, 361 (1985).

[6] M. Dine, L. Randall, and S. D. Thomas, Nucl. Phys. B458, 291 (1996).

[7] M. Fukugita and T. Yanagida, Phys. Lett. B 174, 45 (1986).

[8] A. G. Cohen, D. B. Kaplan, and A. E. Nelson, Annu. Rev. Nucl. Part. Sci. 43, 27 (1993).

[9] Y. Cui, L. Randall, and B. Shuve, J. High Energy Phys. 04 (2012) 075.

[10] D. E. Kaplan, M. A. Luty, and K. M. Zurek, Phys. Rev. D 79, 115016 (2009).

[11] T. Cohen, D. J. Phalen, A. Pierce, and K. M. Zurek, Phys. Rev. D 82, 056001 (2010); J. Shelton and K. M. Zurek, Phys. Rev. D 82, 123512 (2010); M. L. Graesser, I. M. Shoemaker, and L. Vecchi, J. High Energy Phys. 10 (2011) 110; N. F. Bell, K. Petraki, I. M. Shoemaker, and R. R. Volkas, Phys. Rev. D 84, 123505 (2011); C. Cheung and K. M. Zurek, Phys. Rev. D 84, 035007 (2011);
M. L. Graesser, I. M. Shoemaker, and L. Vecchi, arXiv:1107.2666.

[12] D. B. Kaplan, Phys. Rev. Lett. 68, 741 (1992); S. M. Barr, Phys. Rev. D 44, 3062 (1991); S. M. Barr, R.S. Chivukula, and E. Farhi, Phys. Lett. B 241, 387 (1990); M. Fujii and T. Yanagida, Phys. Lett. B 542, 80 (2002); R. Kitano and I. Low, Phys. Rev. D 71, 023510 (2005); R. Kitano, H. Murayama, and M. Ratz, Phys. Lett. B 669, 145 (2008); G. R. Farrar and G. Zaharijas, Phys. Rev. Lett. 96, 041302 (2006); L. Roszkowski and O. Seto, Phys. Rev. Lett. 98, 161304 (2007); O. Seto and M. Yamaguchi, Phys. Rev. D 75, 123506 (2007); J. J. Heckman and S.-J. Rey, J. High Energy Phys. 06 (2011) 120; G. Kane, J. Shao, S. Watson, and H. B. Yu, J. Cosmol. Astropart. Phys. 11 (2011) 012; M. Ibe, S. Matsumoto, and T. T. Yanagida, Phys. Lett. B 708, 112 (2012); B. von Harling, K. Petraki, and R. R. Volkas, arXiv:1201.2200.

[13] A. G. Cohen and D. B. Kaplan, Phys. Lett. B 199, 251 (1987).

[14] M.-z. Li, X.-1. Wang, B. Feng, and X.-m. Zhang, Phys. Rev. D 65, 103511 (2002); A. De Felice, S. Nasri, and M. Trodden, Phys. Rev. D 67, 043509 (2003); M. Yamaguchi, Phys. Rev. D 68, 063507 (2003); R. H. Brandenberger and M. Yamaguchi, Phys. Rev. D 68, 023505 (2003). 
[15] J. March-Russell and M. McCullough, J. Cosmol. Astropart. Phys. 03 (2012) 019.

[16] T. Chiba, F. Takahashi, and M. Yamaguchi, Phys. Rev. Lett. 92, 011301 (2004); F. Takahashi and M. Yamaguchi, Phys. Rev. D 69, 083506 (2004).

[17] T. Gherghetta, C. F. Kolda, and S. P. Martin, Nucl. Phys. B468, 37 (1996); A. Basboll, Int. J. Mod. Phys. A 25, 3375 (2010).

[18] M. Maniatis, Int. J. Mod. Phys. A 25, 3505 (2010).

[19] P. Sikivie, in "The Axion Couplings, " Based upon lectures given at Les Houches Summer School on the Architecture of Fundamental Interactions at Short
Distances, Les Houches, France, 1985 and at the 27th International GIFT Seminar on Cosmology and Particle Physics, Peniscola, Spain, 1986.

[20] A. Dolgov and K. Freese, Phys. Rev. D 51, 2693 (1995); A. Dolgov, K. Freese, R. Rangarajan, and M. Srednicki, Phys. Rev. D 56, 6155 (1997).

[21] A. Anisimov and M. Dine, Nucl. Phys. B619, 729 (2001).

[22] S. Kasuya, M. Kawasaki, and F. Takahashi, Phys. Rev. D 68, 023501 (2003).

[23] K. Kamada, Ph.D. thesis, The University of Tokyo, 2011.

[24] H. Iminniyaz, M. Drees, and X. Chen, J. Cosmol. Astropart. Phys. 07 (2011) 003. 